\title{
PSYCHO-SOCIAL DEPRESSION AND CARDIOVASCULAR HEALTH; A STUDY ON RISK FACTORS IN HYDERABAD, PAKISTAN.
}

\author{
Raza Naseer ${ }^{1 \& 2}$, Muhammad Muneeb ${ }^{1 \& 2}$, Sanjay Maheshwari ${ }^{1 \& 2}$, Asad Khatri $^{1 \& 2}$, Shamoon Noushad ${ }^{1 \& 3} \&$ Sadaf Ahmed $^{1 \& 4}$. \\ Advance Educational Institute \& Research Centre \\ Liaquat University of Medical \& Health Sciences \\ IBMS, Dow University of Health Sciences \\ Dept. of Physiology, University of Karachi
}

\begin{abstract}
Cardiovascular health hazards has become one of major life threats in Pakistan, the risks may vary from province to province. Present study aims to find out the role of psycho-social depression \& its relation with risks of cardiovascular maladies in city of Hyderabad-Pakistan. A cross-sectional study was conducted from November 2012 to February 2013; based on multiple logistic to investigate the relationship between Depression, lifestyle \& blood pressure variations. The Study included 174 subjects both males and females gender with age between $18-25$ years. Depression rating scale was used to evaluate type \& cause of depression furthermore BMI and blood pressure of each subject were also noted. Our study observed that the intensity of stress is directly related with the disturbed blood pressures. Study also observed that with the increasing BMI the prevalence of depression is high. It is therefore concluded that depression is the major risk factor that will increase the risks of cardiovascular maladies; along with other factors like obesity, psychosocial issues like elevating crime \& violence rate could be the major cause of depression in Hyderabad city.
\end{abstract}

\section{KEY WORDS}

Cardiovascular, stress, BMI, depression, blood pressure.

\section{INTRODUCTION}

Over the last decade there has been a marked increase in depression and related psychological disturbances in youth of Pakistan that was investigated in many communities. However the collection of information was always survey based and subjects were evaluated by designing structured questionnaire . Our young populace has been prone to many health hazards as of increased exposure to risk factors and causative factors (Chapman, 2005),specially due to lifestyle and many habitual characteristics there is an increase prevalence of vascular pathologies (Goodman, 2003 \& Davidson, 2001) and these Vascular problems are the leading cause of many cerebrovascular \& cardiovascular diseases(Hayward, 1994). This study investigated the relationship of depressive symptoms, social support, and a range of personal health behaviors in youngsters. Depressive symptoms and psychosocial burden associations can be a link with lack of physical activity, BMI, irregular sleep hours, smoking, low social support etc. (Beck, 1993). Bidirectional causal pathways are likely to link health behaviors with depressed mood. The risk of having vascular problem can be directly related to level of depression (Gurven, 2012 \& Goldston, 2008). Even people whose blood pressures are exactly average for their age have a higher cardiovascular risk than those with lower pressures (WHO, 2003). Any elevation, decline and variation in B.P can be an indicative of a disease of quantity rather than quality (Shahid, 2009). In general, the higher the pressure, the worse the outlook, and again there is no evidence of any dividing line between pressures that carry a low risk and pressures that are associated with heart attacks(Humayun, 2009). Blood pressure tends to rise with advancing age (Kannel, 2000), as prevalence of hypertension in a bit noticeable in south Asian countries (Gurven, 2012). Cardiovascular diseases are also a major global health problem reaching epidemic proportions in Low and middle income countries, including Pakistan, that contribute significantly to the global burden of cardiovascular disease (Jafar, 2003), High risks of cardiovascular disease have been reported in South Asian populations(Shahid, 2009), regardless of whether they live overseas or in their native countries It is speculated that high risk behaviors.

\section{METHODOLOGY}

This study was designed to find out the current prevalence of pre hypertension, depressive moods and lifestyle associations and to bring light on this overly neglected issue. Blood pressure was recorded with Sphygmomanometer (Geneva WHO Expert Committee 2003). Two measurements with 20 minutes interval were taken. Only young adults were included in the study and a sample space of 174 was chosen by stratified sampling technique. A structured questionnaire with multiple logistics criteria was administered to evaluate the symptoms related to sleep disturbances, fears, mood and worries etc.

\section{Inclusion Criteria:}

The targeted population must belong to different localities of Hyderabad city representing different ethnic, socio-economic and educational groups and must be aged between 18 to 25 years.

This process is undertaken by the group of researchers, each being assigned a stratum. Data was analyzed on Microsoft excel and SPSS version 17.0.

\section{RESULTS}

Percents of BMIs among Depression categories 


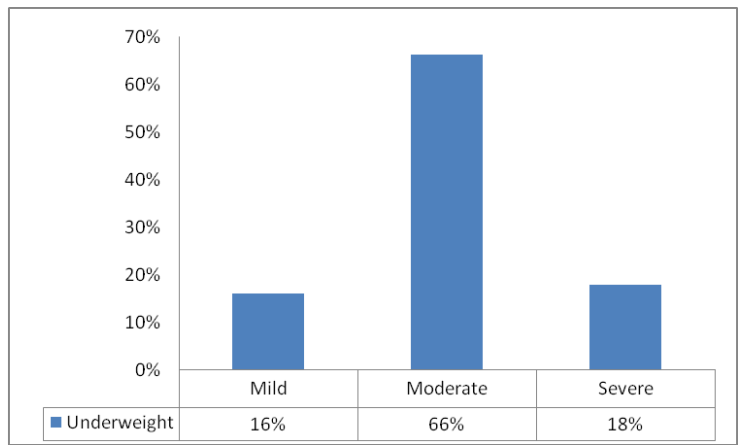

FIGURE\#1: Level of moderate stress is higher among underweight individuals along with almost equal number of individuals in mild and severe cases.

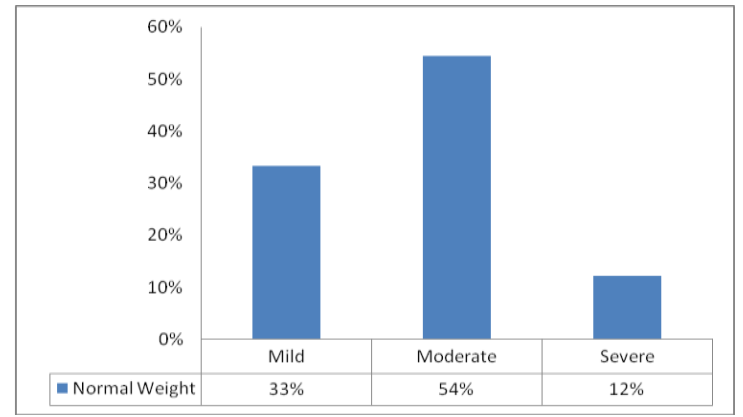

FIGURE\#2: Level of moderate stress is higher among normalweight individuals along with mild stress.

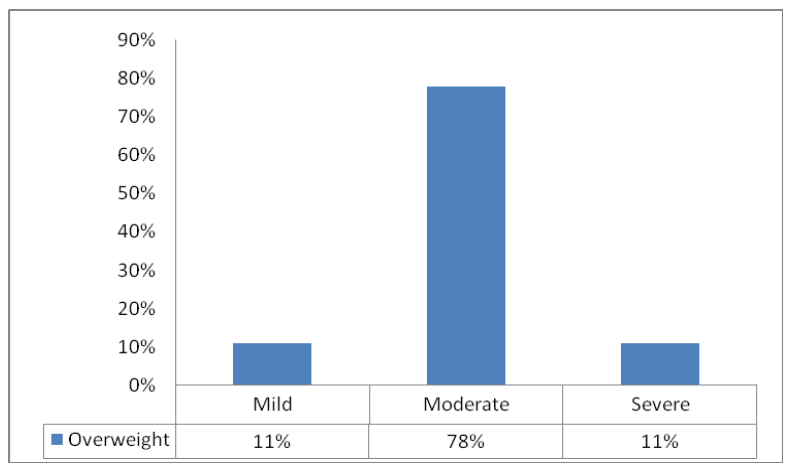

FIGURE\#3: Level of moderate stress is higher among overweight individuals than normal and underweight.

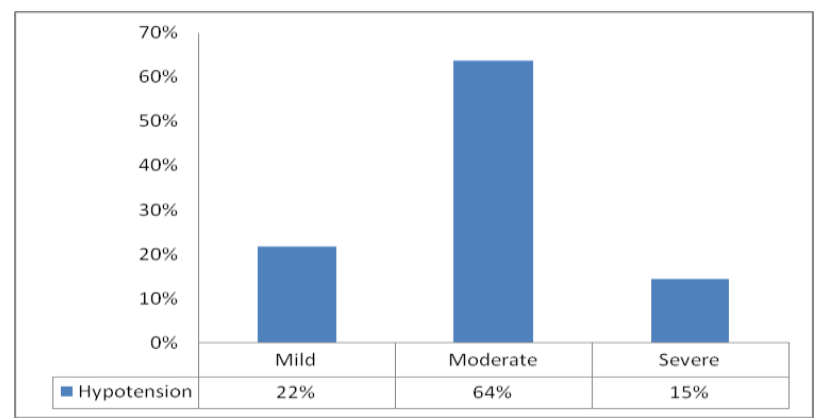

FIGURE\#4: Showing Level of moderate stress is higher along with the hypotension in $64 \%$ when compared with severe and moderate level of stress. 


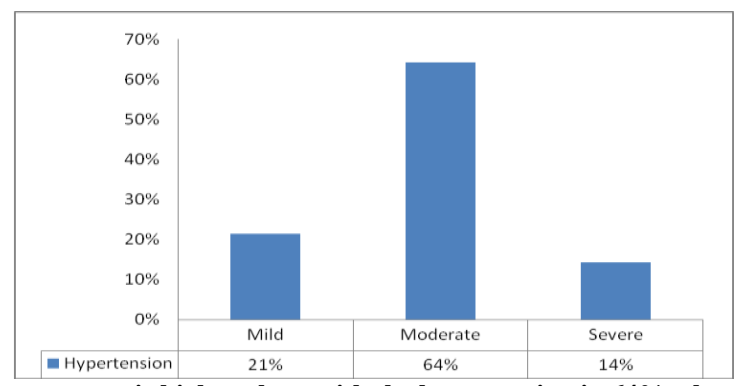

FIGURE\#5: Showing Level of moderate stress is higher along with the hypertension in $64 \%$ when compared with severe and moderate level of stress.

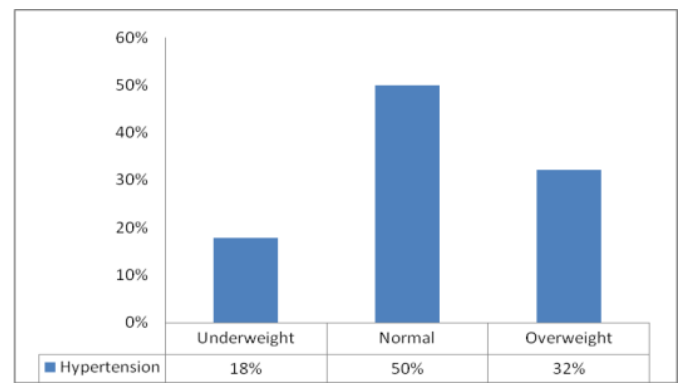

FIGURE\#6: Showing additional information about BMI and prehypertensive values.

The overall results summarized that the variations in blood pressure i.e. pre hypotensive or hypertensive values showed that these individuals might be at cardiovascular risk and the relation with the occurrence of psycho social stress has also been significant. The individuals found to have moderate level of psychosocial stress along with disturbed B.P from mild to severe levels but moderate level of stress was found to be an emerging cause of B.P fluctuations. Moreover the Lifestyle is found to be a correlate of psycho social stress as moderate level of stress is also found in all individuals but mostly overweight individuals reported stress when compared with low or normal weight.

\section{DISCUSSION}

Our results showed that subjects with minor or mild depression are at higher risk of variations in B.P. findings also suggest that this association is not entirely but partially related to predictable risk factors including BMI, psychosocial pressures and level of physical activity. Thus other psychosocial behaviors associated with are likely to be responsible for this relation. Many researchers had investigated about the causes and their effects (Ravaja, 1996) and suggested that adiposity \& weight problems are the major controllable contributor to cardiovascular complications (ref) as well as on a high priority it is a blunt consequence related to suppressed moods, sadness, habits and hopelessness (Johnston, 2004, McElroy, 2004 \& Grundy, 1998). These findings also merit attention towards value of physical exercise, healthy lifestyles and stress free surroundings (Parker, 2007). Another study suggests that BMI is the leading contributor (Licinio, 2003) and socioeconomic status is a weak determinant of cardiovascular risks (Matthews, 2002), therefore we were interested in determining the physical characteristics of cardiovascular functions that are associated with depression (Musselman, 1998) after controlling for potentially confounding variables (Wulsin, 2003). The "vascular depression" hypothesis is also a supporting and related comorbidity of depression, vascular disease, and vascular risk factors as well as the association between symptoms of sadness and related illnesses. Other associations were found between depressive symptoms and skin, respiratory illness, ulcer, hypotension, and diabetes which can be a relative factor in one of many causes of cardiovascular alterations. Standardized assessment of mental disorders by direct interview in which associations between specific physical illnesses and implications for clinical practice and neurobiological research in depression have been discussed in many studies and of much clinical importance. We have examined the public health impact of the psychosocial burden on adolescents' physical and mental health, that is also related with a large proportion of the disease burden within the total population. Despite the growing recognition of the importance of both chronic disease and depressive disorders to the health of individuals and communities, research examining their interrelationship has been the subject of surprisingly little empirical review. Depressive disorders have been associated with risk factors for cardiovascular disease (CVD), such as smoking and physical inactivity (Wulsin, 2003), and mental illness, in general, has been associated with increased mortality due to CVD (WHO, 2003). In general, persons who are depressed are much more likely to develop coronary artery disease. Cognitive behavioral interventions have been useful in managing obesity, largely by modifying eating behaviors and dietary choices in addition to decreasing psychological distress and sedentariness (Hayward, 1994 \& Licinio, 2003). Strikingly, children and adolescents with major depressive disorder appear to manifest an increased risk for subsequently becoming overweight suggesting that both depressive disorders and their treatment are relevant to the prevalence of obesity. The relationship between obesity and depressive disorders thus appears to be reciprocal, with advances in the recognition and treatment of each of these diseases potentially fostering improved mental and physical health(Chapman, 2005). The main objectives of our study were to study risk factor levels and their possible regional differences with the determinants of cardiovascular risk factors, to explore risk factor track, i.e. the maintenance of the relative ranking of an individual with respect to psychological and behavioral risk factors and to collect background information for future intervention strategies carefully measured phenotypes, lifestyle measures and psychosocial burdens background information (Wulsin, 2003). With the vascular measurements and results obtained the study identified the potential of psychosocial burdens and important insights need to be addressed regarding the mechanisms of pre-hypertension, hypotension or depression itself., Though there is a possibility of difference in prevalence but also the factors causing these in different sets of populations. However this study provides a unique opportunity to study the association of psychological disturbance, lifestyle in youth and to relate the lifetime burden of risk factors on 
vascular problems in these subjects. By linking BMI and depression with blood pressure variations as determinants of adult health in this study allowed us the hypothesis that common diseases and disorders have their origin in early life with relation of environment consequences(Goldston, 2008). Over the last decade there has been a marked increase in information on the epidemiology of psychiatric disorders, particularly major depression, in adults living in different communities and in families (Bushra, 2009). Among cardiovascular alterations hypertension is one of the most important life threatening disease (Hashmi, 2003), the cause of hypertension in Pakistan vary province to province. These cardio risks prevalent in most cities across Pakistan and we suppose the condition in Hyderabad would be no different (if not worse). However, the levels of hypertension in Hyderabad, Pakistan are about $18.5 \%$ (6). There are several factors that have been highly associated with the condition includes Smoking, Obesity or being overweight, Diabetes, Sedentary lifestyle, Lack of physical activity, High levels of salt intake (sodium sensitivity) (Adrogué, 2007 \& Miller, 2000). It is thought that it is more prevalent in urban rather than rural areas and is a disease more common in individuals belonging to a higher socio-economic class (Jafar, 2003). Researchers hold different beliefs about which one of the causes is the most important. Our study observed that the intensity of stress is directly related especially with hypertension. Study also observed that with the increasing in age the prevalence of all form of depression was observed. It is therefore concluded that depression is the major risk factor that will increase the risk of hypertension in Pakistan; but along with other factors like obesity and aging, it can lead to CVS and related pathological conditions. Observe psycho-social depression role \& its relation in hypertension in city of Hyderabad-Pakistan. Elevating crime \& violence rate could be the major cause of depression in Hyderabad city. Creating awareness and hence changing the mindset of young adults regarding this problem. The past 2 decades have seen a dramatic increase in factors affecting physical and mental health consequences of traumas, negative events, and chronic strains. Psychosocial burden also account for stress with variability in terms of gender, race, ethnic, age, marital status, and socioeconomic status differences in physical and emotional well-being. This study also hopes to determine the major causes and the associated risks and proposing solutions and lifestyle changes that might improve the current situation.

\section{REFERENCES:}

- Adrogué, H. J., \& Madias, N. E. (2007). Sodium and potassium in the pathogenesis of hypertension. New England Journal of Medicine, 356(19), 1966-1978.

- Beck, A. T., Steer, R. A., Beck, J. S., \& Newman, C. F. (1993). Hopelessness, depression, suicidal ideation, and clinical diagnosis of depression. Suicide and Life-Threatening Behavior, 23(2), 139145 .

- Bushra, R., \& Ali, H. (2009). Surveillance of depression in Karachi, Pakistan.Neurosciences, 14(2), 193-195.

- Chapman, D. P., Perry, G. S., \& Strine, T. W. (2005). PEER REVIEWED: The Vital Link Between Chronic Disease and Depressive Disorders. Preventing chronic disease [electronic resource]., 2(1).

- Davidson, S., Judd, F., Jolley, D., Hocking, B., Thompson, S., \& Hyland, B. (2001). Risk factors for HIV/AIDS and hepatitis C among the chronic mentally ill. Australian and New Zealand Journal of Psychiatry, 35(2), 203-209.

- Goldston, K., \& Baillie, A. J. (2008). Depression and coronary heart disease: a review of the epidemiological evidence, explanatory mechanisms and management approaches. Clinical psychology review, 28(2), 288-306.

- Goodman, E., Slap, G. B., \& Huang, B. (2003). The public health impact of socioeconomic status on adolescent depression and obesity. Journal Information, 93(11).
- Gurven, M., Blackwell, A. D., Rodríguez, D. E., Stieglitz, J., \& Kaplan, H. (2012). Does blood pressure inevitably rise with age? Longitudinal evidence among forager-horti culturalists. Hypertension, 60(1), 25-33.

- Hashmi, S. K., Afridi, M. B., Abbas, K., Sajwani, R. A., Saleheen, D., Frossard, P. M., ... \& Ahmad, U. (2007). Factors associated with adherence to anti-hypertensive treatment in Pakistan. PLoS One, 2(3), e280.

- Hayward, C. (1994). Psychiatric illness and cardiovascular disease risk.Epidemiologic reviews, 17(1), 129-138.

- Humayun, A., Shah, A. S., \& Sultana, R. (2009). Relation of hypertension with body mass index and age in male and female population of Peshwar, Pakistan.J Ayub Med Coll Abbottabad, 21(3), 63-65.

- Jafar, T. H., Levey, A. S., Jafary, F. H., White, F., Gul, A., Rahbar, M. H., \& Chaturvedi, N. (2003). Ethnic subgroup differences in hypertension in Pakistan.Journal of hypertension, 21(5), 905-912.

- Johnston, E., Johnson, S., McLeod, P., \& Johnston, M. (2004). The relation of body mass index to depressive symptoms. Canadian Journal of Public Health,95(3), 179-183.

- Kannel, W. B. (2000). Elevated systolic blood pressure as a cardiovascular risk factor. The American journal of cardiology, 85(2), 251-255.

- Lewington S et al., (2002) Prospective Studies Collaboration. Age-specific relevance of usual blood pressure to vascular mortality: a metaanalysis of individual data for one million adults in 61 prospective studies. Lancet.;360(9349):1903-1913.

- Licinio, J., \& Wong, M. L. (2003). The interface of obesity and depression: risk factors for the metabolic. Revista Brasileira de Psiquiatria, 25(4), 196-197.

- Matthews, K. A., Kiefe, C. I., Lewis, C. E., Liu, K., Sidney, S., \& Yunis, C. (2002). Socioeconomic trajectories and incident hypertension in a biracial cohort of young adults. Hypertension, 39(3), 772-776..

- McElroy, S. L., Kotwal, R., Malhotra, S., Nelson, E. B., Keck, P. E., \& Nemeroff, C. B. (2004). Are mood disorders and obesity related? A review for the mental health professional. J Clin Psychiatry, 65(5), 634-651.

- Miller, G. D., DiRienzo, D. D., Reusser, M. E., \& McCarron, D. A. (2000). Benefits of dairy product consumption on blood pressure in humans: a summary of the biomedical literature. Journal of the american College of Nutrition, 19(sup2), 147S-164S..

- Musselman, D. L., Evans, D. L., \& Nemeroff, C. B. (1998). The relationship of depression to cardiovascular disease: epidemiology, biology, and treatment.Archives of general psychiatry, 55(7), 580.

- Parker, E. D., Schmitz, K. H., Jacobs Jr, D. R., Dengel, D. R., \& Schreiner, P. J. (2007). Physical activity in young adults and incident hypertension over 15 years of follow-up: the CARDIA study. American journal of public health, 97(4), 703-709.

- Ravaja, N., Keltikangas-Järvinen, L., \& Keskivaara, P. (1996). Type A factors as predictors of changes in the metabolic syndrome precursors in adolescents and young adults: A 3-year follow-up study. Health Psychology, 15(1), 18.

- S M Grundy (March 1998) Multifactorial causation of obesity: implications for prevention. Am J Clin Nutr; vol. 67 no. 3:563S$572 \mathrm{~S}$.

- Shahid A., Asad R. K. \& Shazia A. (2009) Disease Burden of Ischemic Heart Disease in Pakistan and its Risk Factors. Ann. Pak. Inst. Med. Sci.; 5(3): 145-150

- Tunstall-Pedoe H, ed. (for the WHO MONICA Project) MONICA Monograph and Multimedia Sourcebook.World largest study of heart disease, stroke, risk factors and population trends. 19792002. Geneva, World Health Organization, 2003.

- Weissman, M M. (1987) Advances in psychiatric epidemiology: rates and risks for major depression. American Journal of Public Health April: Vol. 77, No. 4, pp. 445-451. 
- Wulsin, L. R., \& Singal, B. M. (2003). Do depressive symptoms increase the risk for the onset of coronary disease? A systematic quantitative review.Psychosomatic Medicine, 65(2), 201-210. 